\begin{tabular}{|c|c|c|c|c|c|c|c|}
\hline \multirow{3}{*}{18.23 März 19} & \multirow[b]{2}{*}{ Siern $6^{r}$ Gr. } & \multirow{2}{*}{\multicolumn{2}{|c|}{ Eintr. $40^{\circ} \mathrm{N}$}} & Sternzeit. & $\begin{array}{l}\text { Mittl. Speir. } \\
\text { Zeit. }\end{array}$ & \multirow{2}{*}{$\underbrace{\begin{array}{c}\text { Beobacht. } \\
\text { sicher auf }\end{array}}_{0,3}$} & \multirow{2}{*}{$\underbrace{\begin{array}{c}\text { Zeitbest. } \\
\text { sicher auf }\end{array}}$} \\
\hline & & & & $10^{h} 7^{\prime} 37^{\prime \prime}, 8$ & $10^{\mathrm{h}} 21^{\prime} 7^{\prime}, 3$ & & \\
\hline & 8 & Eintr. & $5 \mathrm{~N}$ & $\begin{array}{lll}10 & 10 & 23,3\end{array}$ & 102352,3 & 2,0 & \\
\hline & 8 & Eintr. & $45 \mathrm{~N}$ & 103148,7 & $1045 \quad 14,3$ & 0,5 & \\
\hline April 14 & 7 & Eintr. & $70 \mathrm{~N}$ & $\begin{array}{lll}10 & 9 & 20,9\end{array}$ & $840 \quad 36,5$. & 0,2 & 0,5 \\
\hline 15 & 8 & Eintr. & $20 \mathrm{~S}$ & $11 \quad 1 \quad 14,4$ & 92825,6 & 0,5 & 0,3 \\
\hline 19 & 6. $7 \mathrm{r}$ & Eintr. & $3 \mathrm{~N}$ & 113135,8 & $942 \quad 58,4$ & 0,2 & 1,0 \\
\hline May 18 & 69 leunis & Eintr. & $40 S$ & 132334,0 & $940 \quad 36,7$ & 0,2 & 0,3 \\
\hline Aug. 3 & $6 r$ & Eintr. & $45 \mathrm{~N}$ & $\begin{array}{lll}23 & 14 & 3,0\end{array}$ & 142644,0 & 3,0 & 0,3 \\
\hline & & Austr. & $40 \mathrm{~N}$ & $23 \quad 4957,1$ & 15232,3 & 0,5 & $\longrightarrow$ \\
\hline Oct. 10 & 7. 8 & Eintr. & $\mathbf{0}$ & $\begin{array}{lll}20 & 7 & 2,5\end{array}$ & $\begin{array}{lll}6 & 52 & 52,2\end{array}$ & 1,0 & 0,3 \\
\hline Nov. 10 & 7 & Eintr. & $25 \mathrm{~N}$ & $\begin{array}{lll}22 & 13 & 33,4\end{array}$ & $\begin{array}{lll}6 & 57 & 9,1\end{array}$ & 0,3 & 0,5 \\
\hline 13 & 6 & Eintr. & $40 \mathrm{~S}$ & $\begin{array}{lll}22 & 42 & 25,9\end{array}$ & $\begin{array}{lll}714 & 9,2\end{array}$ & 0,3 & 3,0 \\
\hline 21 & $\delta$ geminorum & Einlr. & $8 \mathrm{~N}$ & $\begin{array}{l}04523,6 \\
\end{array}$ & $\begin{array}{lll}8 & 45 & 19,4\end{array}$ & 1,5 & 0,3 \\
\hline & & Austr. & $5 \mathrm{~N}$ & 14024,0 & $\begin{array}{lll}9 & 40 & 10,9\end{array}$ & 1,0 & - \\
\hline 1824 Juny 2 & . . . . & Eintr. & $45 \mathrm{~S}$ & 142626,1 & $941 \quad 20,9$ & 0,2 & 2,0 \\
\hline July 23 & ...$\cdot \cdot$ & Eintr. & $85 \mathrm{~N}$ & $2325 \quad 18,0$ & $1518 \quad 13,2$ & 0,5 & 0,5 \\
\hline & 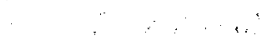 & Austr. & $75 N$ & 233531,0 & 152825,6 & 3,0 & 一 \\
\hline Aug. 5 & $6^{r}$ & Eintr. & $10 \mathrm{~S}$ & $20 \quad 9 \quad 46,6$ & $11 \quad 127,0$ & 2,0 & 2,0 \\
\hline Nov. 29 & $8 \mathrm{ki}$ piscium & Eintr. & $40 S$ & 0 $38 \quad 22,4$ & $\begin{array}{lll}8 & 3 & 53,3\end{array}$ & 0,3 & 2,0 \\
\hline Dec. 26 & 7. 8 & Eints. & $10 \mathrm{~N}$ & 04846,1 & $628 \quad 5,6$ & 0,5 & 0,3 \\
\hline
\end{tabular}

Schwerd.

$Z u$ den in Speier, 1823 und 1824, beobachteten Bedechungen.

1823 . Januar 30.

q Virginis scheinbare Länge $189^{\circ} 1^{\prime} 34^{\prime \prime}, 4$; Breite - $5^{\circ} 19^{\prime} 46^{\prime \prime}, 3$; Conjunction in wahrer Speierer Zeit in der Länge: aus dem Eintr. 17 $41^{\prime} 41^{\prime \prime}, 6+1,65 d B$

woraus $d B=+2^{\prime \prime}, 06$

$$
\text { Austr. } \quad 51,7-3,25 d B
$$

und die verbesserte Conjunction $1741^{\prime} 45,0$

zu Paris nach der conn. des tems 1717 18,8

$$
2426,2
$$

nur um $1 ", 2$ von der bekannten Länge verschieden, welches, mit stündlicher Bewregung $32^{\prime} 43^{\prime \prime}, 0$, die Correction - 0",65 gibt.

\section{März 19.}

Für den ersten Eintritt ist der scheinbare Mondsort AR. $89^{\circ} 5^{\prime}$; Decl. $+26^{\circ} 0^{\prime}$. Den Stern vermochte ich nicht aufzufinden.

Für die vier folgenden Eintritte sind nach der Ordnung die Sterne: hist. cél. p. 310 . 5 $56^{\prime} 27^{\prime \prime}, 5$ trois, Fil; $5^{\text {h }} 58^{\prime} 0^{\prime \prime}, 5 ; 5^{\text {h }} 58^{\prime} 17^{\prime \prime} ; 5^{\text {h }} 58^{\prime} 52^{\prime \prime}$.
An demselben Tage hat Herr Prof. Hallaschka in Prag eilf Eintritte beobachtet - Astr. Nachr. Nr. 37. Für den vierten ist der Stern hist. cél. p. 145. $5^{\text {h }} 53^{\prime} 14^{\prime \prime}$ trois. Fil $=$ p. 310 . $5^{\mathrm{h}} 52^{\prime} 14^{\prime \prime} ;$ der achte stimmt mit Speier II, der zehnte mit Speier III, der eilfte mit Speier IV überein; für die übrigen habe ich keine Sterne gefunden; die scheinbaren Mondsörter für selbige sind, der Ordnung nach, $88^{\circ} 3^{\prime}+26^{\circ} 7^{\prime} ; 88^{\circ} 13^{\prime}+26^{\circ} 6^{\prime}$; $88^{\circ} 17^{\prime}+26^{\circ} 5^{\prime} ; 88^{\circ} 30^{\prime}+26^{\circ} 3^{\prime} ; 89^{\circ} 5^{\prime}+25^{\circ} 58^{\prime} ;$ $89^{\circ} 11^{\prime}+25^{\circ} 57^{\prime} ; 89^{\circ} 21^{\prime}+25^{\circ} 55^{\prime}$. Noch finde ich an diesem Tage einen Eintritt von Herrn Prof. David in Prag beobachtet. - Bode Jahrh. 1827. S. 116 - es ist derselbe Stern wie Hall. IV.

Die Culmination des Mondes ist an diesem Tage beobachtet in Greenwich Nr. 63. der Astr. Nachr.

$$
\text { April } 14 .
$$

Der Stern ist hist. cél. p. 204. $4^{\text {h }} 33^{\prime} 35^{\prime \prime}, 8$; dessen Eintritt ist zugleich in Marseille - conn. d. t. 1827. p. 265 - und in Viviers - corresp. astr. IX, 276 beobachlet Forden; anch hat an demselben Tage Herr 
Prof. Hallaschka in Prag zwei Eintrilte beobachlet Astron. Nachr, Nr. 37. - zu denen ich die \$terue nicht finde; die scheinbaren Mondsörter sind $68^{\circ} 14^{\prime}+25^{\circ} 25^{\prime}$ und $68^{\circ} 15^{\prime}$ mit derselben Abweichung. Die Culmination ist in Mailand beobachtet. Efemeridi 1825. p. 49.

$$
\text { April } 15 .
$$

Der Stern ist hist. cél. p. 145. $5^{\text {h }} 41^{\prime} 40^{\prime \prime}$ Irois. Fil. Ein nahgelegener Stern $-5^{\text {h }} 38^{\prime} 24^{\prime \prime}-$ ist an demselben Tage in Marseille - conn. d. t. 1827. p. 265 - und in Viviers - corresp. astron. IX, 276 - bedeckt worden. Auch hat Herr. Prof. Bessel in Königsberg an diesem Tage den Eintritt eines Sterns achter Gröfse beobachtet - Bode Jahrb. 1827. S. 196 - den jedoch weder ich in den Verzeichnissen, noch Herr Prof. Schwerd am Himmel, haben auffinden können; der einzige, zu dem scheinbaren Mondorle $84^{\circ} 23^{\prime}+25^{\circ} 49^{\prime}$ passende, schien dem letzteren 10-11 Gröfse zu seyn. Die Culmination ist in Mailand beobachlet - Efem. 1825 p. 69 - wobei ich bemerke, dafs dacelbst an diesem und dem folgenden Tage der Halbmesser auf dem Parallel um $10^{\prime \prime}$ bis $12^{\prime \prime}$ zu klein angegeben ist; auch ist in der hist. cél. p. 145 bei 132 tauri $4 G^{\prime \prime}$ slatl $47^{\prime \prime}$ zu lesen.

\section{April 19.}

Den zu dieser Bedeckung gehörenden Stern finde ich nirgends; der scheinbare Monlort ist $144^{\circ} 14^{\prime}+11^{\circ} 43^{\prime}$; die Culmination an diesem Tage ist beolbachtet in Berlin Jahrb. 1827: S. 148 -; Dorpat - Astron. Nachr. No.40; Leipzig - Nr. 56; Königsberg - Nr. 59; Greenwich Nr. 63.

\section{Mai 18.}

Zu dem, was über diesen Eintrilt in Nr. 60 der Asir. Nachr. bemerkt worden, finde ich noch die Königsberger Beobachlung - Jahrb. 18.27 S. 196 - nachzutragen; sie giel,t, nach denselben Elementen berechnet, die Lünge $1^{\text {h }} 12^{\prime} 44^{\prime \prime}, 9-1,5 d D+2,0 d A K$; es ist aber aus Nr. 60 $d D=+2 ", 4$ und die Königsberger Culminalion desselben Tages gilit $d D=+2^{\prime \prime}, 2$. wie $d A R=-2^{\prime \prime}, 7$; Jelzteres wird nach der Mailänder Culmination $=-3^{\prime \prime}, 2$; aus dem Mitlel dieser Werthe folgt die Iaänge $1^{\text {th }} 12^{\prime} 35^{\prime \prime}, 4$. Die Greenwicher Culmination gibt $d A R=-3^{\prime \prime}, 9$. Astr. Nachr. Nr.63, wo an. diesem Tage statt $\eta$ Virg, zal lesen ist nVirg. und am $15^{\text {ten }}$ October K Capric. slatt des exsten K Aquarii.

$$
\text { A u g. } 3 .
$$

Der bedeckle Stern ist hist. cél. p. 145. 6h $27^{\prime} 17^{\prime \prime}, 5$ $=$ p. 316. $6^{\mathrm{h}} 27^{\prime} 18^{\prime \prime}$ tr. Fil ; diese Beobachtungen, mit- telst P. VI, 165 und 204 reducirt, 'stimmen in der AR. nicht überein; der mittlere Ort für 1800 wird aus der ersteren $96^{\circ} 48^{\prime} 46^{\prime \prime},,^{9}+24^{\circ} 45^{\prime} 33^{\prime \prime}, 4$; aus der zweiten $96^{\circ} 48^{\prime} 26^{\prime \prime}, 1+24^{\circ} 45^{\prime} 30^{\prime \prime}, 3$; auch die beiden Reductionen in der Com. d. t. VII. p. 387 und IX. p. 413 sind verschieden; nach der ersteren ist der Stern als 57 geminorum in Bode's grofen Catalog übergegangen. Lege ich das Mittel aus meinen obigen Angaben zum Grunde, so ergilst sich die Conj. in AR. und wahrer Zeit in Speier aus dem Eintritte $15^{\text {h }} 41^{\prime} 13^{\prime \prime} 1+1,406 d D$

$$
\text { Austritte } \quad 31,2-1,531 d D
$$

woraus $d D=+6^{\prime \prime} 163$ u. die verb.Conj. $1541^{\prime} 21,8$

für Paris nach der Conn. des terns 151653,4

$2428,4+1,47 d A R$.

\section{October 10.}

Der Stern ist hist. cél. p. 567. 18 8 $^{\prime} 15^{\prime \prime}, 2=$ la Caille coelum austr. Zona XXIV. p. sup. $18^{\text {h }} 4^{\prime} 28^{\prime \prime}$; die dortigen 78

Reductionstafeln geben den Ort für 1750: $271^{\circ} 25^{\prime} 25^{\prime \prime}, 7$ - $26^{\circ} 9^{\prime} 47^{\prime \prime}, 4$; la Lande giltt ihn für 1790 in der Conn. d. t. XIII. $272^{\circ} 2^{\prime} 33^{\prime \prime}-26^{\circ} 9^{\prime} 27^{\prime \prime}$. Der Eintritt desselben Sterns ist in Marseille beobachtet - conn. d. $t$. 1827. p. 267 - und in Prag von Herrn Prof. Bittner. Jahrb. 1827. S. 117.

Novbr. 10.

Den Ort dieses Sterns hat zuerst Herr Prof. Harding für 1800 angegeben in der Monatl. Corr. XXVIII, 313: $317^{\circ} 11^{\prime} 48^{\prime \prime}, 8-14^{\circ} 51^{\prime} 11^{\prime \prime}, 5$; in der Folge hat ihn auch Herr Prof. Bessel in der 117ten Zone beobachtet, wonach der Ort für 1825 wird: $317^{\circ} 32^{\prime} 52^{\prime \prime}: 6-14^{\circ}$ $45^{\prime} 18^{\prime \prime}, 9$, woraus eine merkliche eigene bewegung folgt. Die Culmination ist an diesem Tage in Mailand - Efem. 1825. p. 56 - und in Paris - Astr. Nachr. Nr. 48 beobachtet.

Novbr. 13.

Der Slern ist 15 pisc. und dessen Eintritt anch in Abo beobachtet - Astr. Nachr. Nr. 57. Die Astronomen in Florenz haben diese Bedeckung nicht angezeigt; die Culmination in Paris - Astr. Nachr. Nr. 48; Königsberg - Nr. 59; Greenwich Nr. 63.

\section{Novbr. 21.}

Conjunclion in AR. und wahrer Zeit des Beobachiungsortes: 
Speier Eintritt $10^{\mathrm{h}} 32^{\prime} 26^{\prime \prime}, 7-0,042 d D \quad$ Länge

Austritt $103225,8-0,279 d D \quad\left(24^{\prime} 25^{\prime \prime}\right)$

Prag (H.) Eintritt 10.56 23,0 - 0,139dD 4821,3

Prag (B.) Eintritt $1056 \quad 21,5 \quad 48 \quad 19,8$

Viviers Austritt $101723,6+0,022 d D \quad 922,8$

Marseille Austritt $102010,3+0,084 d D \quad 12 \quad 9,5$

Trient Eintritt $104245,9-0,331 d D \quad 3444,2$

-Austritt $104314,9+0,236$ a $D \quad 3514,1$

Mit Ausnahme von Trient sind dies die bekannten Längen; der erwähnte Ort hat besunderes Unglück in der Längenbestimmung, wie man aus den divergirenden Resultaten der Herrn Wurm und Santini sehen'kann. Astr. Nachr. Nr. 34. Corr. Astr. VHI, 211. Obige mit Speier correspondirende Beobachlungen finden sich im Jahrb. 18.27. S. 117. 128; in den Corr. Astr. XI, 127; in der Conn. d. t. 1827 , p. 268 ; in den Efemeridi 1825. p. 8.

$$
\text { 1824. Jun. } 2 .
$$

An demselben Tage sind zwei Eintritte ron Herm Prof. Hallaschka in Prag - Astr. Nachr. Nr,65 - und zwei in Lübeck - Nachr. 62. - beobachtet worden.

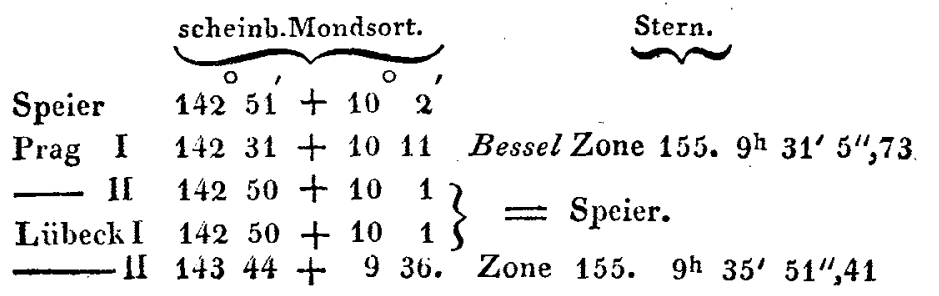

$=$ hist. cél. p. 324. $9^{\mathrm{h}} 33^{\prime} 59^{\prime \prime}$. in den Astr. Nachr. a. a. O. wird der lefzte Stern nach der Conn. d. t. angegeben; es muls jodoch daselbst statt p. 248 gelesen werden 244.

Jul. 23.

Der Stern ist 9 geminorum.

$$
\text { A ug. } 5 .
$$

Der Siern ist hist. cél. p. $566.18^{\text {h }} 1^{\prime} 5^{\prime \prime}, 5$ tr. Fil. Die Culmination ist an diesem Tage von Herrn Professor David in Prag beobachlet. Astr. Nachr. Nr. 64.

$$
\text { Decbr. } 26 .
$$

Der Stern ist hist. cél. p. $130.22^{\mathrm{h}} 57^{\prime} 58^{\alpha}, 5=$ p. 184 $22^{\text {h }} 57^{\prime} 39^{\prime \prime}=$ Conn. d. 1. X. p. 452 . $344^{\circ} 24^{\prime} 14^{\prime \prime}$. Er kounnt auch in der $112^{\text {ten }}$ Zone des Herrn. Prof. Bessel vor, wonach der mittlere Ort für 1825: $344^{\circ} 51^{\prime} 30^{\prime \prime}, 8$ - $1^{\circ} 26^{\prime} 37^{\prime \prime}, 5$; scheinbarer Ort für die Zeit des Eintrilts: $344^{\circ} 51^{\prime} 39^{\prime \prime}, 0-1^{\circ} 26^{\prime} 32^{\prime \prime}, 2$; correspondirende Beobachtungen: von Herm Professor David in Prag Astron. Nachr. Nr. 71; von mir in Mannheim Eintrilt $6^{\text {h }} 28^{\prime} 20^{\prime \prime}: 2$ mittlere Zeit. Es ergibt sich hieraus die Conj. in gerader Aufsteigung und wahrer Zeit des Beobachtungsortes

$$
\begin{aligned}
& \text { für Speier um }{ }^{h} 17^{\prime} 13,5+1,643 d D \\
& \text { - Mannh. }-61723,8+1,695 d D \\
& \text { - Prag - } 64111,0+1,381 d D
\end{aligned}
$$

Es ist offenbar, dafs die hieraus alzzuleitende Länge meiner Wohnung nicht in Uebereinstinumung mit dem bekannten Meridian-Unterschiede von Speier $(7 ", 0)$ zu bringen ist: der Fehler liegt in der Zeitbestimmung, indea ich lange zuvor keine correspondirende Höhen genommen hatte.

\section{Heiligenstein.}

Sternbedeckungen, in Mannheim beobachtet.

\section{1825. März 22 .}

Eintritt eines Sterns $9^{\mathrm{r}}$ Gröfse um $7^{\text {h }} 20^{4} 30^{\prime \prime}, 6$ wahrer Zeit. Der Stern ist hist. cél. p.192. $2^{\mathrm{h}} 5^{\prime} 55^{\prime \prime}$ trois. fil, und sein miltlerer Ort für 1800: $31^{\circ} 31^{\prime \prime} 30^{\prime \prime}, 4+16^{\circ} 22^{\prime} 51^{\prime \prime}, 1$; reducirt durch 8 und 29 Arietis.

II. M̈̈r $\mathbf{z} 26$.

Eintritt eines Sterns $\mathbf{3}^{\mathrm{r}}$ Gröfse um $7^{\text {h }} 44^{\prime} 14^{\prime \prime}, 6$ wahrer Zeit; dieselbe Beobachtung hat Herr Prof. Schwerd in Speier um $8^{\text {h }} 5^{\prime} 56^{\text {" }}, 9$ Sternzeit gemacht; der Stern ist hist. cél. p. 315. $5^{\text {h }} 39^{\prime} 37^{\prime \prime}$ treis. fll. und tein mill. Ort für 1800: $84^{\circ} 53^{\prime} 14^{\prime \prime}, 2+22^{\circ} 50^{\prime} 48^{\prime \prime}, 1$ : bei der Reduction habe ich bemerkt, dafs daselbst die Zenithdistanz von 216 Mayeri um etwa anderthalb Minuten zu südlich angegeben ist gegen die übereinstiwenenden liesultate ron
Länge $24^{\prime} 32^{\prime}$

Piazzi und Barry (z. 354): auch ist am drillen Faden stalt $5^{\text {h }} 39^{\prime} 45^{\prime \prime}$ zu lesen: $35^{\prime \prime}$.

die Conj. in Speier ergilts sich un $7^{\mathrm{h}} 31^{\prime} 55^{\prime \prime}, 9+0,363 d D$ in Mannheim $73150,6+0,378$

$$
-5,3
$$

diese Meridiandifferenz sollte $+\mathbf{7}^{\prime \prime}, 0$ seyn.

An demselben Tage haben die Herren Nicolai und Schwerd den correspondirenden Eintritt eines Sterns $7^{\text {ter }}$ Grölse beobachtet jener um $9^{\text {h }} 57^{\prime} 16^{\prime \prime}, 2$, dieser um $9^{\text {h }} 57^{\prime} 38^{\prime \prime}, 3$ beides Sternzeit. Da dieser Stern nirgends vorkommt, so hat $11 \mathrm{r}$. $S$. am $8^{\text {ten }}$ April ihn mit 140 tauri am Kreismicromete a verslichen; hiernach wird sein scheinbarer Ort für die Bedeckung $86^{\circ} 5^{\prime} 11,6+22^{\circ} 25^{\prime} 36^{\prime \prime}, 9$ 